Provided for non-commercial research and education use. Not for reproduction, distribution or commercial use.

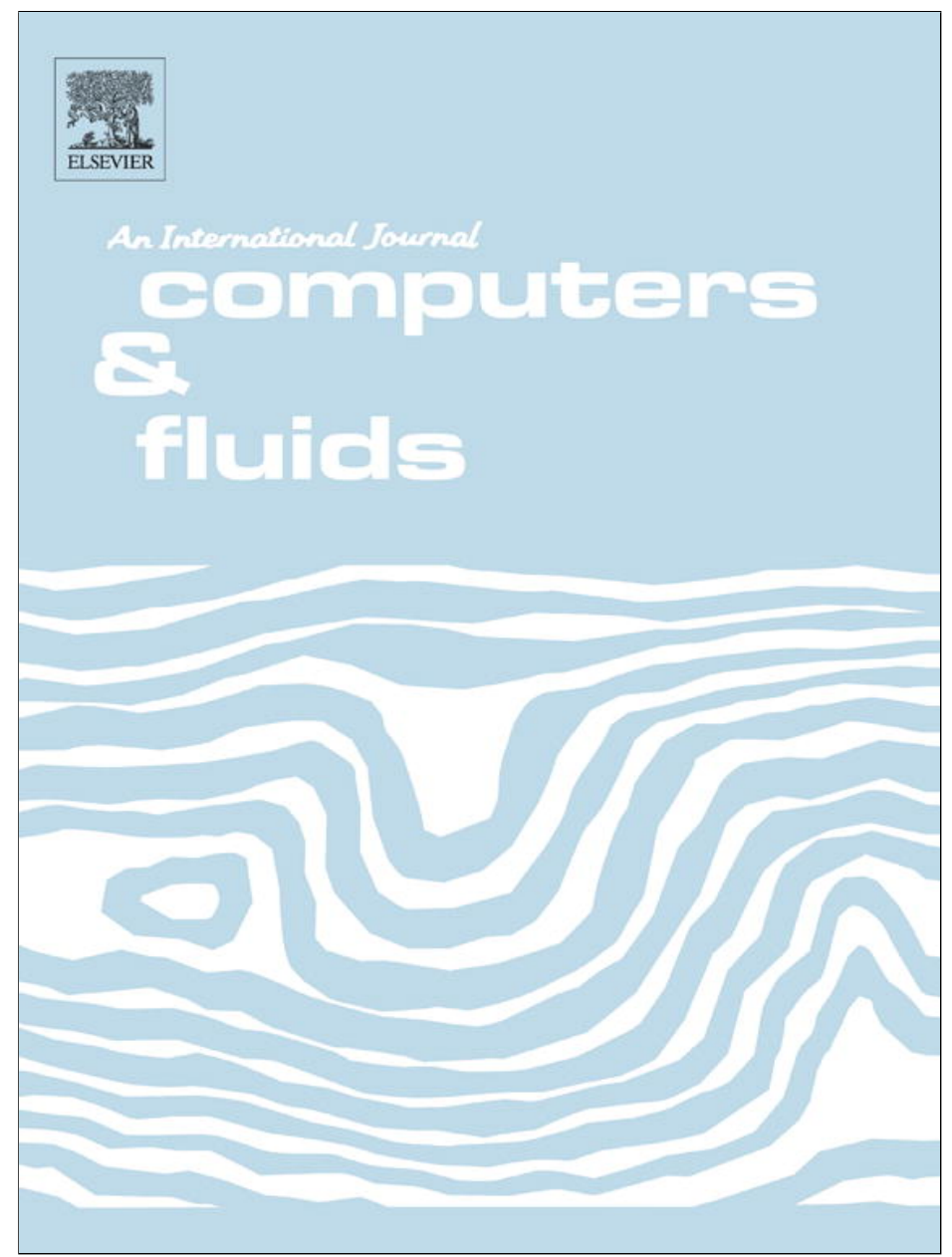

(This is a sample cover image for this issue. The actual cover is not yet available at this time.)

This article appeared in a journal published by Elsevier. The attached copy is furnished to the author for internal non-commercial research and education use, including for instruction at the authors institution and sharing with colleagues.

Other uses, including reproduction and distribution, or selling or licensing copies, or posting to personal, institutional or third party websites are prohibited.

In most cases authors are permitted to post their version of the article (e.g. in Word or Tex form) to their personal website or institutional repository. Authors requiring further information regarding Elsevier's archiving and manuscript policies are encouraged to visit:

http://www.elsevier.com/copyright 


\title{
Interface boundary conditions in near-wall turbulence modeling
}

\author{
S.V. Utyuzhnikov \\ School of Mechanical, Aerospace E Civil Engineering, Manchester University, Manchester M13 9PL, UK \\ Moscow Institute of Physics \& Technology, Dolgoprudny 141700, Russia
}

\section{A R T I C L E I N F O}

\section{Article history:}

Received 21 October 2011

Received in revised form 14 June 2012

Accepted 31 July 2012

Available online 15 August 2012

\section{Keywords:}

Domain decomposition

Interface boundary condition

Wall functions

Turbulence

Low-Reynolds-number model

$k-\epsilon$ Model

\begin{abstract}
A B S T R A C T
Near-wall turbulence modeling is a very computationally expensive problem. A new approach suggested here allows us to avoid calculations of the region with high gradients in the vicinity of the wall while retaining sufficient overall accuracy. A non-overlapping domain decomposition is introduced in application to low-Reynolds number RANS models. The domain decomposition is achieved via the transfer of the boundary condition from the wall to an interface boundary. If the governing equations in the inner domain are simplified, then the transmission (interface) boundary conditions are of Robin type. These boundary conditions can be obtained in an analytical form despite the fact that they are nonlinear. Possible ways to achieve a reasonable trade-off between efficiency and accuracy are discussed. The obtained interface boundary conditions are mesh-independent. They can be used to avoid the computationally expensive resolution of a high-gradient region near the wall. Moreover, once the solution is constructed in the outer domain, the near-wall profile can be restored if required. In two extreme cases, if the interface boundary is too close to the wall or too far from it, the so-constructed solution to the problem automatically corresponds to low- and high-Reynolds number models, respectively. It is shown that the new interface boundary conditions are uniformly applicable in a wide range of the interface boundary locations.
\end{abstract}

(c) 2012 Elsevier Ltd. All rights reserved.

\section{Introduction}

It is well known that the problem of near-wall turbulence modeling is very computationally expensive. In the vicinity of the wall, there exists a thin laminar sublayer due to both the no-slip boundary condition for the velocity and blocking effect caused by the wall. Because this sublayer has a significant influence on the flow structure, its accurate resolution is of great importance. The structure of the layer is highly variable and can be described by low-Reynolds number (LR) RANS models. Sufficiently accurate resolution of the laminar sublayer requires a very fine mesh. As a consequence, even though the thickness of the sublayer is only about $1 \%$ of the entire thickness of the boundary layer, it takes up to $90 \%$ of computational time [1]. Thereby, the application of LR to industrial problems is often prohibitively expensive even on the modern computers. That is especially relevant to multidisciplinary optimization problems where the fluid dynamics module is one of many others and multiple iterations between different modules are required.

In contrast to LR models, high Reynolds number (HR) models do not provide any resolution of the laminar sublayer. Physically, this means that they are formulated outside the sublayer. Thereby,

* Address: School of Mechanical, Aerospace \& Civil Engineering, Manchester University, Manchester M13 9PL, UK.

E-mail address: s.utyuzhnikov@manchester.ac.uk mathematically, they require new boundary conditions because the original boundary conditions at the wall are no longer applicable in straightforward manner. This problem is usually resolved via implementation of Dirichlet boundary conditions called wall functions. In the original form, they represented an analytical solution obtained for a thin plate $[2,3]$. This idea soon became very popular especially within industrial community because of its efficiency and reasonable accuracy. In most cases wall functions are semiempirical and have some free parameters to be selected for special classes of problems. In addition, the solution is often mesh dependent that does not give much confidence in the obtained results unless additional calculations are carried out. As follows from the main idea of wall functions, this approach should meet serious problems if the first mesh node/cell is situated inside the viscous sublayer because of the contradiction between the model assumptions and real physics.

Increasing demands to the accuracy and efficiency from industrial and academic communities encourage researches to seek ways to derive more universal wall functions. The scalable wall functions were suggested in [4] to reduce the mesh dependence. As shown in [5], the pressure gradient must be taken into account to avoid mesh dependence. However, as demonstrated in [6], this requirement is only applicable to standard wall functions based on the log-law profile assumption. It appears that the adaptive wall functions [6] are mesh independent although they are obtained for zero pressure gradient flows. An interesting idea to take into 
account source terms, such as pressure gradient and buoyancy force, has been realized in the numerical and analytical wall functions $[7,8]$. They are based on a solution obtained in a subgrid area related to the nearest wall cell either numerically or analytically. In contrast to the standard wall functions, the obtained solution is incorporated into the numerical approximation used in the core flow domain. The analytical wall functions are based on the assumption of a piece-wise linear viscosity profile. The numerical wall functions are free from this assumption. However, they are significantly more computationally expensive as compared to the traditional wall functions.

Instead of constructing a solution for the first mesh node, it was suggested in [9] to transfer the boundary conditions from the wall to an interface boundary, which might correspond to the first node. For a model equation, it was demonstrated that, although the exact solution cannot be obtained in the near-wall subdomain without the solution in the entire domain, the boundary conditions can. The piece-wise linear approximation, which is used in the analytical wall functions, is exploited to derive an interface boundary condition (IBC) for HR models in [10]. The obtained boundary conditions are of Robin type and called the Robin type wall functions in [11] although the title of IBC would be more appropriate here. As shown in [10], the boundary conditions (wall functions) are mesh independent and do not contain free parameters. Their application to some test cases in $[10,11]$ demonstrated that the achieved accuracy is reasonable and the solution is not too sensitive to the position of the interface boundary. As noted in [11], the solution obtained via the analytical and Robin-type wall functions should be intrinsically the same provided that they are based on the same assumptions. However, the levels of robustness and convergence are different. This issue is discussed in Section 5 in more detail.

In [11], it was shown that the IBCs [10] can be obtained via the theory of Calderón-Ryaben'kii's potentials [12] applied to a onedimensional case. More generally, this leads to nonlocal IBCs or nonlocal wall functions. As demonstrated in [13] for a two-dimensional model equation, the nonlocal IBCs can be important in the case of complex geometries. This approach can be alternative to other wall-distance-free turbulence models (see, e.g., [14-16]).

In the current paper, the technique of the boundary condition transfer is applied to LR model. In contrast to the previous works, the turbulent viscosity profile is approximated nonlinearly. This leads to nonlinear boundary conditions of Robin type. Once the solution is obtained in the outer domain, the appropriate solution in the inner domain can also be found. Thus, the approach can be interpreted as a non-overlapping domain decomposition applied to LR model.

The rest of the paper is organized as follows. In Section 2, the non-overlapping domain decomposition approach is described for a linear problem. Then, it is extended to nonlinear formulations in Section 3, where nonlinear IBCs are derived. Possible implementations of the IBCs to RANS equations are described in Section 4. In Section 5, Chien's LR $k-\epsilon$ model is considered as an example, and the entire algorithm is described in detail. Computational results are given for the channel flow. They demonstrate that the obtained IBCs are uniformly applicable in a wide range of the interface boundary locations. It is also indicated that in extreme cases of the interface boundary situated in the laminar sublayer and far away from it the solution automatically corresponds to the LR (single-domain) and HR models, respectively.

\section{Non-overlapping domain decomposition: Linear case}

Let us first consider the following one-dimensional model equation:

$L_{y} U=f$ defined in an interval $\Omega:=\left[0, y_{e}\right]$ with a Dirichlet boundary condition on the right-hand side:

$l_{y} U(0)=l_{0}$,
$U\left(y_{e}\right)=l_{1}$

Here, $l_{y}$ and $L_{y}$ are linear differential operators of first and second order, respectively. In particular, $l_{y}$ is equivalent to 1 for a Dirichlet boundary condition and derivative $\frac{d}{d y}$ for a Newman boundary condition.

Eq. (1) represents the general form of the boundary-layer-type equation. The right-hand side $f$ is an appropriate source term including, e.g., the pressure gradient in the momentum equation.

Along with the domain $\Omega$, let us introduce domain $\Omega^{-}: \Omega^{-}:=[0$, $\delta$ ], $0<\delta<y_{e}$. Thus, the original domain $\Omega$ is decomposed into two subdomains $\Omega^{-}$and $\Omega^{+}$, where $\Omega^{+}:=\left[\delta, y_{e}\right]$.

To construct a nonoverlapping domain decomposition we introduce two auxiliary BVPs formulated in the domain $\Omega^{-}$.

$1^{0}$.

BVP 1 is formulated for the homogeneous equation:

$L_{y} V=0$,

$l_{y} V(0)=0$,

$V(\delta)=1$.

$2^{0}$.

In turn, BVP 2 reads as follows:

$L_{y} W=f$,
$l_{y} W(0)=l_{0}$,
$W(\delta)=0$.

One can see that

$U=V U(\delta)+W$.

Thus, we arrive at the interface boundary condition formulated at point $y=\delta$ :

$U^{\prime}=V^{\prime} U+W^{\prime}$,

and this boundary condition is of Robin type.

It is worth noting that $V^{\prime} U$ is equivalent to the Steklov-Poincaré operator $S$ determined on $\Omega^{-}$and applied to $U(\delta): S U(\delta):=V^{\prime} U(\delta)$.

IBC (6) can be interpreted as the transmissive boundary condition exactly transferred from the boundary $y=0$ to the interface boundary $y=\delta$, via the theory of potentials [12] one can prove that IBC (6) does not depend on the boundary condition at the boundary $y=y_{e}$ and the position of this boundary itself. Moreover, the operator $L_{y}$ can change in $\Omega^{+}$under some general requirements. Such a domain decomposition is non-overlapping. It is also called the substructuring decomposition, which on a discrete level is reduced to the Schur complement method [17].

Thus, we can use the interface (or transmissive) boundary condition for a wide range of BVPs corresponding to quite different boundaries $y_{e}$ and variations of the right-hand side in the domain $\Omega^{+}$. As soon as we obtain the solution in the domain $\Omega^{+}$, we can restore the solution in the domain $\Omega^{-}$straightforward from (5). It is important to note that, although one cannot obtain the solution in the domain $\Omega^{-}$without the remaining part of the original domain, we are able to obtain IBC.

\section{Non-overlapping domain decomposition: Non-linear case}

Consider a domain decomposition for a non-linear equation:

$\mathcal{L}_{y}(U)=f$,

where $\mathcal{L}_{y}$ is a non-linear differential operator of second order, and boundary conditions (2). 
There are different ways to tackle this problem, via nonlinear potentials introduced in [18], one can obtain transmissive boundary condition at the interface boundary:

$U_{y}=\Psi(U, f)$.

However, such a boundary condition is nonlinear and its computational efficiency is questionable.

One possible algorithm to simplify computations is as follows. First, we can approximate $f$ by a constant in the domain $\Omega^{-}$. Then, solving BVP (7) and (2) with $l_{1}=U$, we obtain $U_{y}(\delta)$. This procedure is equivalent to the identification of the nonlinear Steklov-Poincaré operator $\Psi$ in (8). Then, the results can be stored for different $U$ and $f$ in look-up tables. In some sense, a similar procedure was used in [6] to obtain dependence between $U$ and $\tau_{w}$ for zero pressure gradient flows $(f \equiv 0)$.

Another approaches can be related with approximation of operator $\mathcal{L}_{y}$ by a linear operator $L_{y}$ :

$\mathcal{L}_{y}(U) \approx L_{y} U$.

Then, Eq. (6) yields the IBC for the nonlinear BVP formulated in the domain $\Omega^{+}$.

Such an approach is accompanied by an inevitable error. To reduce this error, we can try to make the approximation more accurate by using an iterative procedure:

$\mathcal{L}_{y}\left(U^{(n+1)}\right) \approx L_{y}^{(n)} U^{(n+1)}$.

Then, we arrive at the following IBC at iteration $n+1$ for the nonlinear BVP set in the outer domain $\Omega^{+}$:

$U^{(n+1) \prime}=V^{(n) \prime} U^{(n+1)}+W^{(n) \prime}$,

where functions $V^{(n)}$ and $W^{(n)}$ are obtained from the following two auxiliary BVPs:

$L_{y}^{(n)} V^{(n)}=0$,

$l_{y} V^{(n)}(0)=0$,

$V^{(n)}(\delta)=1$,

and

$L_{y}^{(n)} W^{(n)}=f^{(n)}$,

$l_{y} W^{(n)}(0)=l_{0}$,

$W^{(n)}(\delta)=0$.

In this way, in particular, we can reach the exact domain decomposition organizing appropriate iterations and specifying the operator $L_{y}$. General efficient iterative algorithms can be found, for example, in [17].

\section{Interface boundary conditions for RANS equations}

Any of the RANS equations besides the continuity one is of second order and has the following structure:

$\left(\mu \phi_{y}\right)_{y}=R_{h}$,

where $\phi$ is the appropriate leading variable of the equation. The right-hand side $R_{h}$ includes the convective and force terms. In general, these terms depend on $\phi, \phi_{y}$, other dependent variables and their derivatives including derivatives along other space directions.

\subsection{One-dimensional domain decomposition}

For the sake of simplicity, first consider a one-dimensional problem. Then, there are different ways for either exact or approximate domain decompositions.

\subsubsection{Exact domain decomposition}

In this case, we linearize the right-hand side $R_{h}$ with respect to both $\phi^{(n)}$ and $\phi_{y}^{(n)}$ :

$R_{h}\left(\phi^{(n+1)}, \phi_{y}^{(n+1)}\right) \approx a^{(n)} \phi_{y}^{(n+1)}+b^{(n)} \phi^{(n+1)}+c^{(n)}$,

where $a^{(n)}, b^{(n)}, c^{(n)}$ are the appropriate linearization coefficients.

Thus, we can introduce a linear operator

$L_{y}^{(n)}:=\frac{\partial}{\partial y} \mu^{(n)} \frac{\partial}{\partial y}-a^{(n)} \frac{\partial}{\partial y}-b^{(n)}$.

Then, the iterative algorithm can be organized in three steps as follows.

Step $1^{0}$

Suppose that we know the field $\phi^{(n)}$ along with its derivative either from an initial approximation or previous iterations. Then, solving BVPs (10) and (11) with $f^{(n)}=c^{(n)}$, we can specify the IBC for the outer domain $\Omega^{+}$:

$\phi^{(n+1) \prime}=V^{(n) \prime} \phi^{(n+1)}(\delta)+W^{(n) \prime}$.

Step $2^{0}$

Upon obtaining the solution in the outer domain, we restore the solution in the inner domain $\Omega^{-}$from

$\phi^{(n+1)}=V^{(n)} \phi^{(n+1)}(\delta)+W^{(n)}$.

Step $3^{0}$

Finally, we can update the transport coefficient $\mu$ in the inner domain and repeat iterations if required.

If convergent, such an algorithm is accurate in the one-dimensional case. Its main advantage is that we can implement completely different meshes in the domains $\Omega^{+}$and $\Omega^{-}$. In addition, the computations in these domains, including the solutions of auxiliary BVPs, can be easily parallelized.

\subsubsection{Approximate domain decomposition}

In the vicinity of a wall, the right-hand side $R_{h}$ can be simplified. One possible way is to either omit or approximate the convective terms. Then, we can obtain the IBC explicitly via a Robin boundary condition:

$\phi^{\prime}=\frac{\phi}{I_{1}}+\frac{I_{1} I_{S 1}-I_{S 2}}{\mu^{*} I_{1}}$,

where $I_{1}=\int_{0}^{y^{*}} \frac{\mu^{*}}{\mu} d y, I_{S 1}=\int_{0}^{y^{*}} R_{h} d y, I_{S 2}=\int_{0}^{y^{*}} \frac{\mu^{*}}{\mu} \int_{0}^{y \prime} R_{h} d y d y^{\prime}$.

Assuming $R_{h}$ is constant, obviously this boundary condition can be reduced to

$\phi^{\prime}=\frac{\phi}{I_{1}}+\frac{R_{h}}{\mu^{*}} \frac{y^{*} I_{1}-\bar{I}_{2}}{I_{1}}$,

where $\bar{I}_{2}=\int_{0}^{y^{*}} \frac{y^{\prime} \mu^{*}}{\mu} d y^{\prime}$.

Such boundary conditions were suggested in $[10,11]$ for highReynolds number models. However, they could also be used as the IBC for low-Reynolds number RANS models.

In $[10,11]$, the transport coefficient $\mu$ was approximated by a piece-wise linear function similar to the analytical wall function approach [7]. In this case, the inner domain can be completely separated from the outer domain. Although the results obtained for the HR models are reasonably good, the accuracy of this approach can be improved if applied to the LR models with a more accurate approximation for the transport coefficient $\mu$.

In the next Section, we apply the following approximation for the turbulent viscosity profile suggested in $[20,21]$ :

$\mu_{t}=\mu_{l} \kappa y^{+}\left(1-\exp \left(-y^{+} / A\right)\right)^{2}$,

where $\kappa=0.41, A=19, y^{+}=u_{\tau} \rho y / \mu_{l}, u_{\tau}=\sqrt{\tau_{w} / \rho}, \tau_{w}=\mu_{l} u_{y}(0), u$ is the velocity, $\mu_{l}$ the laminar viscosity coefficient, $\rho$ density. 
In contrast to the linear profile, $\mu_{t}$ in (18) depends on $\tau_{w}$. Obviously, it is linked with $u_{y}(\delta)$ :

$\tau_{w}=\left(\mu_{l}+\mu_{t}(\delta)\right) u_{y}(\delta)-\int_{0}^{\delta} R_{h u} d y$,

where $R_{h u}$ corresponds to $R_{h}$ in (12) applicable to the momentum equation.

Thereby, the IBC (16) is nonlinear and represents a simplified form of the nonlinear IBC (8) although it is not exact. Practically, it should be realized via an iterative procedure as was demonstrated in the next Section.

\section{Interface boundary conditions for low-Re model}

The application of the IBCs is illustrated on the example of LR $k-$ $\epsilon$ models.

The following five-step iterative algorithm can be applied.

Step $1^{0}$.

Assume we know $\tau_{w}$ and $\epsilon$ from either an initial approximation or previous iteration. Then, we set the boundary condition for $u$ at $y=\delta$ from (17):

$u^{\prime}=\frac{u}{I_{1}}+\frac{P_{x}}{\mu^{*}} \frac{y^{*} I_{1}-\bar{I}_{2}}{I_{1}}$,

where $\bar{I}_{2}=\int_{0}^{y^{*}} \frac{y^{\prime} \mu^{*}}{\mu} d y^{\prime}, \mu=\mu_{l}+\mu_{t}, \mu_{t}$ is determined by (18), $P_{x}$ is the approximation of the pressure gradient at $y=\delta$.

Step $2^{0}$.

For the turbulent kinetic energy we set Dirichlet boundary condition:

$k(\delta)=\sqrt{\tilde{\epsilon} \mu_{t}\left(y^{*}\right) /\left(C_{\mu} \rho f_{\mu}\right)}$.

Here,

$\mu_{t}=C_{\mu} \rho f_{\mu} k^{2} / \tilde{\epsilon}$

$\tilde{\epsilon}(y)=\epsilon-\frac{2 v k}{y^{2}}$

$v=\mu_{l} / \rho$,

$f_{\mu}=f_{\mu}\left(y^{+}\right)$.

\section{Step $3^{0}$.} [7]:

For $\epsilon$, we can use the following Dirichlet boundary condition

$\epsilon(y)=\max \left\{\frac{k^{3 / 2}}{C_{l} y}, \frac{2 v k}{y^{2}}\right\}$,

where $C_{l}=2.55$.

Step $4^{0}$.

For all other functions, the IBCs are represented by (16).

Step $5^{0}$.

Finally, $\tau_{w}$ is updated via Eq. (19).

Upon the solution in the outer domain has been obtained, the solution in the inner domain can be calculated straightforward if required.

As can be seen, iterations between the inner and outer domains are needed. Thus, IBC (20) can be interpreted as a simplified version of the IBC (8). However, the solution in the inner domain is not required and the recalculation of the IBC is relatively fast.

\subsection{Test cases}

The domain decomposition approach described in Section 5 has been tested in application to a fully developed plane channel flow.

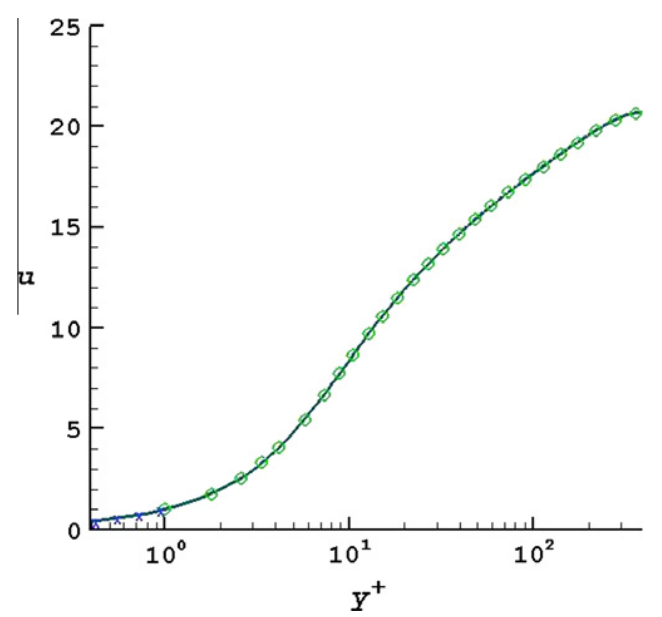

Fig. 1. Velocity profile: $R e_{\tau}=395$. Solid line is single block solution; $\times$ corresponds to inner profile; $o$, outer profile.

As an example, the LR model by Chien [19] has been chosen as the governing equations:

$\frac{\partial}{\partial y}\left[\left(v+v_{t}\right) \frac{\partial u}{\partial y}\right]=p_{x} / \rho$,

$v_{t}\left(\frac{\partial u}{\partial y}\right)^{2}-\epsilon+\frac{\partial}{\partial y}\left[\left(v+v_{t} / \operatorname{Pr}_{k}\right) \frac{\partial k}{\partial y}\right]=0$,

$C_{\epsilon 1} f_{1} \frac{\tilde{\epsilon}}{k} v_{t}\left(\frac{\partial u}{\partial y}\right)^{2}-C_{\epsilon 2} f_{2} \frac{\tilde{\epsilon}^{2}}{k}+E+\frac{\partial}{\partial y}\left[\left(v+v_{t} / \operatorname{Pr}_{\epsilon}\right) \frac{\partial \tilde{\epsilon}}{\partial y}\right]=0$,

$\epsilon=\epsilon_{0}+\tilde{\epsilon}, \quad v_{t}=C_{\mu} f_{\mu} k^{2} / \epsilon, \quad f_{\mu}=1-e^{-0.0115 y^{+}}$,

$f_{1}=1, \quad f_{2}=1-0.22 e^{\left(-R e_{T} / 6\right)^{2}}, \quad C_{1}=1.35, \quad C_{2}=1.8$,

$\epsilon_{0}=2 v \frac{k}{y^{2}}, \quad E=-2 v \frac{\tilde{\epsilon}}{y^{2}} e^{-y^{+} / 2}, \quad \operatorname{Re}_{t}=\frac{k^{2}}{\tilde{\epsilon} v}$.

Similar to [9], two regimes have been considered: $R e_{\tau}=395$ and $R e_{\tau}=3950$. Here, the Reynolds number $R e_{\tau}$ is defined via the friction velocity $u_{\tau}$ and the half of the channel height: $R e_{\tau}=u_{\tau} h / v$.

In all computations, first the solution in the outer domain is obtained and then it is restored in the inner domain. As to the interface boundary $\delta$, different values of $y^{+*}=\delta u_{\tau} / v$ are considered. The results are compared against a single block solution obtained on a mesh fine enough to reach a mesh independent solution. In addition, the algorithm from Section 5 has been applied to the HR $k-$ $\epsilon$ model. This approach is equivalent to the algorithm based on the Robin-type wall functions [11] with the nonlinear approximation (18) for the viscosity coefficient. Such a type of the solution is called NRWF below. In this case, the solution is not obtained in the inner domain.

In Fig. 1 , the velocity profile is given for $y^{+*}=1$ and $R e_{\tau}=395$. As can be seen, the two-block solution is very close to the benchmark solution. In the case of $y^{+*}=50$ shown in Fig. 2, there is some discrepancy with the single block solution mostly in the outer domain. The NRWF approach gives notably different friction coefficient, which is associated with the inclination of the profile $u$. As $\delta$ increases, the error in the outer domain is gradually reduced. However, the solution in the inner domain retains to be quite accurate as can been seen in Fig. 3 for $y^{+*}=100$. For this case and greater values of $\delta$, the wall-function based solution NRWF almost coincides with the LR two-block solution.

In the case of $R e_{\tau}=3950$, the two-block solution for $y^{+*}=1$ almost coincides with the benchmark solution similar to $R e_{\tau}=395$ as shown in Fig. 4. However, for $y^{+*}=10$ (Fig. 5) the difference between the two solutions is more visible. It is important to note that the wall-function based solution NRWF gives a significant error. 


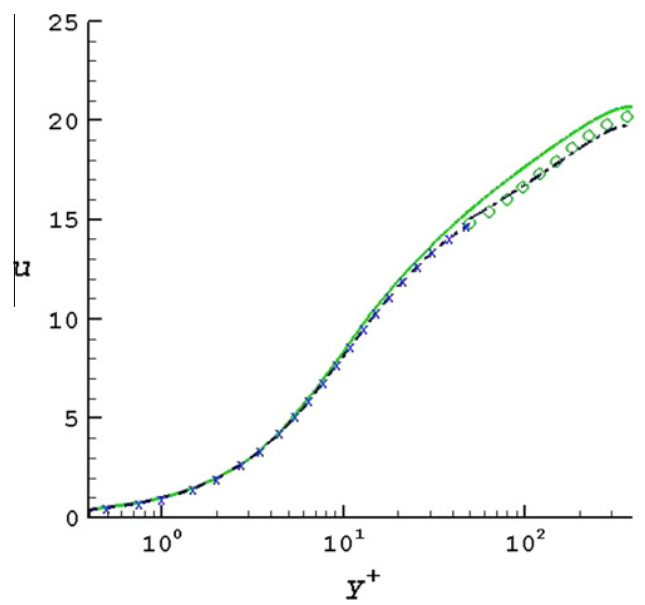

Fig. 2. Velocity profile: $R e_{\tau}=395$. Solid line is single block solution; $\times$ corresponds to inner profile; $o$, outer profile; dash-dotted line, wall-function solution.

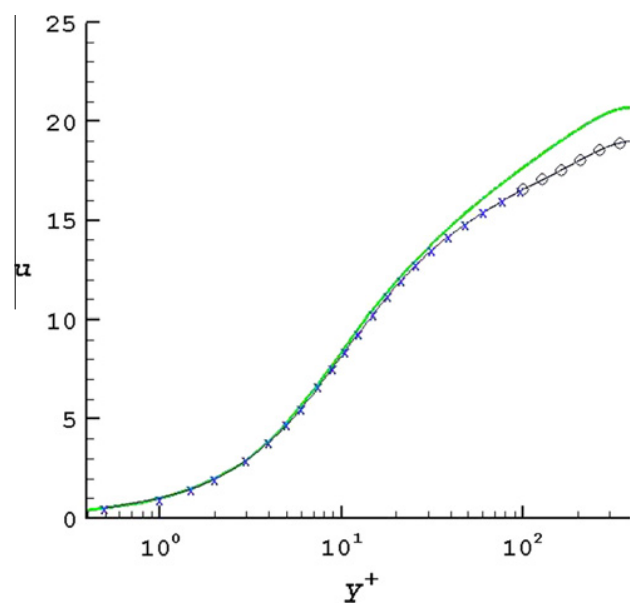

Fig. 3. Velocity profile: $R e_{\tau}=395$. Solid line is single block solution; $\times$ corresponds to inner profile; $o$, outer profile.

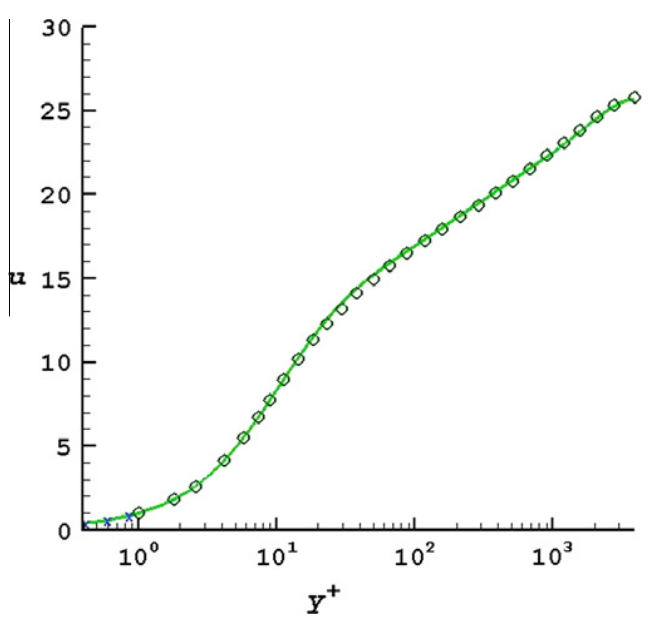

Fig. 4. Velocity profile: $R e_{\tau}=3950$. Solid line is single block solution; $\times$ corresponds to inner profile; $o$, outer profile.

It is interesting to note that if $\delta$ increases then the two-block solution is very close to the benchmark solution even for $y^{+*}=200$ as demonstrated in Fig. 6. The HR solution with NRWF almost coincides with the LR two-block solution in the outer domain.

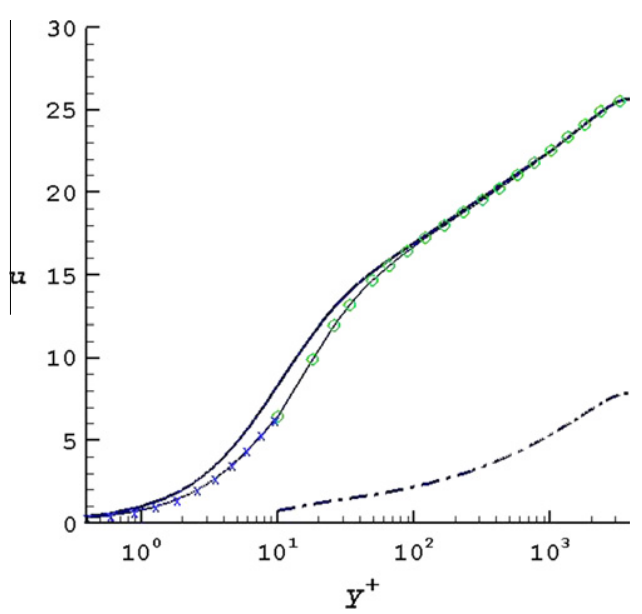

Fig. 5. Velocity profile: $R e_{\tau}=3950$. Solid line is single block solution; $\times$ corresponds to inner profile; $o$, outer profile; dash-dotted line, wall-function solution.

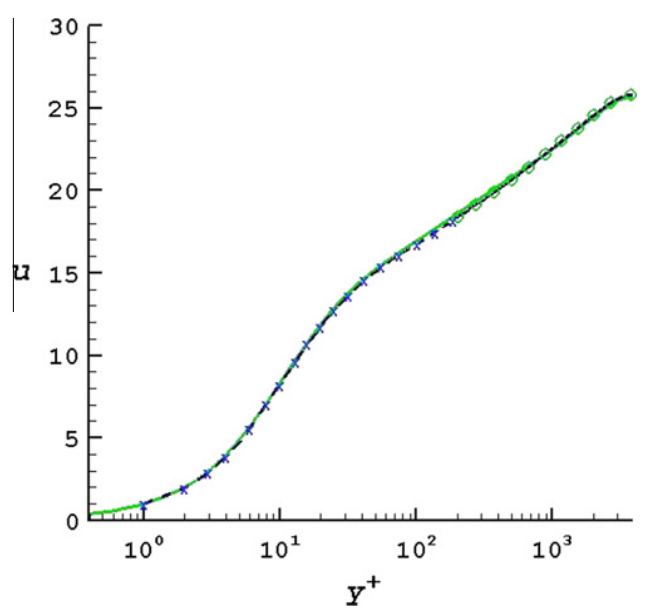

Fig. 6. Velocity profile: $R e_{\tau}=3950$. Solid line is single block solution; $\times$ corresponds to inner profile; $o$, outer profile.

Overall, the HR solution is significantly more accurate in comparison to the approach based on the piece-wise approximation of the viscosity profile $\mu_{t}$ described in [9].

It is worth noting here that the domain decomposition described in Section 4.1.1 gives the solution coinciding with the benchmark solution because it is accurate in one-dimensional case. However, it is more time consuming and the algorithm is more complicated.

\subsection{Comparison against analytical and numerical wall functions}

It can be interesting to compare the IBC approach against analytical wall function (AWF) and numerical wall function (NWF) algorithms. Although the latter two techniques were developed for HR models, with some modifications they could also be applied for LR models. Both these algorithms are mesh dependent while the IBCs are formulated in a mesh independent form.

The AWF were derived for a piece-wise linear profile of the turbulent viscosity $\mu_{t}$ in the inner domain. It is important that this approach leads to a Dirichlet boundary condition at point $\delta$, which is obtained via integration of the governing equation in a simplified form. This boundary condition (wall function) obviously depends on the boundary condition at the wall. As we know, the wall boundary condition can be transferred to the interface boundary. 
Eventually, the AWF can be reformulated in the differential form similar to (6) as a linear combination of the function and its derivative. The solutions based on AWF and IBC would coincide provided that the same approximations for the governing equation (including profile $\mu_{t}$ ) were used since the solution to the problem must be unique. However, the realization of AWF is reduced to

$U^{(n) \prime}=V^{(n) \prime} U^{(n+1)}+W^{(n) \prime}$

in contrast to IBC (14) because of the Dirichlet boundary condition. Thus, one of the two leading terms in the boundary condition (6) is taken from the previous iteration that inevitably affects the convergence.

In turn, if applicable to a LR model, the NWF would be similar to the one-dimensional exact domain decomposition described in Section 4.1.1. However, this approach would lead to a Dirichlet boundary condition similar to AWF and its realization would be less efficient because one of the two leading terms was taken from the previous iteration. In addition, it is well known that Robin boundary conditions are more robust than Dirichlet or Newman boundary conditions.

\subsection{Extension to a multidimensional case}

IBC (16) can immediately be used for multidimensional problems. However, in this case the right-hand side in Eq. (12) depends on derivatives with respect to the other independent variables. One possible way to overcome this problem is to either neglect or approximate these derivatives using the solution at the interface boundary (see, e.g., $[7,11]$ ). In this case, we obtain locally onedimensional IBC and introduce an inevitable error. In particular, the one-dimensional exact domain decomposition approach described in Section 4.1.1 cannot be exact in a multidimesnional case. A possible improvement over this approach can be based on the combination of the nonlocal domain decomposition [13] with the IBC derived in this paper for one-dimensional case.

\section{Conclusion}

The approach to a non-overlapping domain decomposition for near-wall turbulence modeling has been suggested and analyzed. It is applicable to LR and HR turbulence models and based on transferring the boundary conditions from the wall to an interface boundary. The algorithm allows to avoid computationally expensive calculations related to the near-wall area while retaining a sufficiently high accuracy.

The approach provides interface boundary conditions uniformly applicable for a wide range of interface boundaries. It appears that if at the interface point $y^{+}<1$, then the solution corresponds to the LR model used. In turn, if the interface point $y^{+}>100$, then the solution in the outer domain coincides with the HR solution. Although the former case is accurate, the domain decomposition does not bring any essential advantage because of a negligibly small inner domain. The latter case is intrinsically equivalent to the application of the Robin-type wall functions based on the nonlinear approximation of the turbulent viscosity profile. This leads to a significant improvement in comparison to the piece-wise linear approximation considered in the previous works.
The uniform suitability of the IBC for very different locations of the interface boundary is very important because it yields a flexibility to the approach especially for multidimensional problems. Overall, the interface boundary position needs to be chosen reasonably. If it is too close to the wall, there is little effect from the domain decomposition. In turn, if the boundary is too far from the wall, it affects the accuracy.

As the next step, the developed approach will be applied to multidimensional problems. There are two opportunities. The simplest one can be based on a locally one-dimensional approximation of the governing equations in the near-wall subdomain including approximations of some derivatives along the wall. Intrinsically, it does not distinguish from the approach described in this paper. Another opportunity can be based on the combination of the nonlocal domain decomposition [13] derived for a linear model with the current approach. As demonstrated in [13], the latter approach is significantly more accurate in the case of complex geometries.

\section{References}

[1] Kopp T. On grid-independence of RANS predictions for aerodynamic flows using model-consistent universal wall-functions. In: Proceedings of ECCOMAS CFD'2006. The Netherlands; 2006.

[2] Patankar SV, Spalding DB. Heat and mass transfer in boundary layers. London: Morgan-Grampian Press; 1967.

[3] Launder BE, Spalding BI. The numerical computation of turbulent flows. Comput Methods Appl Mech Eng 1974;3:269-89.

[4] Grotjans H, Menter FR. Wall functions for industrial applications. In: Papailiou KD, editor. Proceedings of computational fluid Dynamics'98, ECCOMAS, vol. 1. Chichester, UK: Springer; 1998. p. 1112-7.

[5] Wilcox DC., Wall matching, a rational alternative to wall functions AIAA Paper 89-611. Reno, NV; 1989.

[6] Kalitzin G, Medic G, Iaccarino G, Durbin PA. Near-wall behavior of RANS turbulence models and implications for wall functions. J Comput Phys 2005;204(1):265-91.

[7] Craft TJ, Gerasimov AV, Iacovides H, Launder BE. Progress in the generalization of wall-functions treatments. Heat Fluid Flow 2002;23(2):148-60.

[8] Craft T], Gant SE, Iacovides H, Launder BE. A new wall function strategy for complex turbulent flows. Numer Heat Transfer 2004;45:301-18.

[9] Utyuzhnikov SV. Some new approaches to building and implementation of wall-functions for modeling of near-wall turbulent flows. Comput Fluids 2005;34(7):771-84.

[10] Utyuzhnikov SV. The method of boundary condition transfer in application to modeling near-wall turbulent flows. Int J Comput Fluids 2006;35(10):1193-204.

[11] Utyuzhnikov SV. Robin-type wall functions and their numerical implementation. J Appl Numer Math 2008;58(10):1521-33.

[12] Utyuzhnikov SV. Generalized Calderón-Ryaben'kii's potentials. IMA J Appl Math 2009;74(1):128-48.

[13] Utyuzhnikov SV. Domain decomposition for near-wall turbulent flows. Int J Comput Fluids 2009;38(9):1710-7.

[14] Drikakis D, Goldberg U. Wall-distance-free turbulence models applied to incompressible flows. Int J Comput Fluid Dyn 1998;10:241-53.

[15] Durbin PA. Near-wall turbulence closure modelling without damping functions. J Theor Comput Fluid Dyn 1991;3(1):113.

[16] Laurence DR, Uribe JC, Utyuzhnikov SV. A robust formulation of the v2-f model. Int J Flow Turbul Combust 2004;73(3):169-85.

[17] Smith BF, Bjøstad PE, Gropp WD. Domain decomposition: parallel multilevel methods for elliptic partial differential equations. Cambridge University Press; 1996.

[18] Utyuzhnikov SV. Nonlinear problem of active sound control. J Comput Appl Math 2010;234(1):215-23.

[19] Chien K-Y. Predictions of channel and boundary-layer flows with a lowReynolds-number turbulent model. AIAA 1982;20(1):33-8.

[20] Cabot W, Moin P. Approximate wall boundary conditions in the large-eddy simulation of high Reynolds number flow. Flow Turbul Combust 1999;63:269-91.

[21] Wang M, Moin P. Dynamic wall modeling for large-eddy simulation of complex turbulent flows. Phys Fluids 2002;14(7):2043-51. 